\title{
Acknowledgment of reviewers who served in 2008
}

(c) The Japanese Society for Hygiene 2008

We greatly appreciate the valuable contributions of the following individuals ${ }^{1}$ who served as reviewers for articles submitted to Environmental Health and Preventive Medicine in 2008.

Akiba, Suminori (Kagoshima)

Arisawa, Kokichi (Tokushima)

Babazono, Akira (Fukuoka)

Endo, Ginji (Osaka)

Harada, Noriaki (Yamaguchi)

Hata, Akira (Chiba)

Hayakawa, Kazuo (Suita)

Himeno, Seiichiro (Tokushima)

Hiramatsu, Kazufumi (Oita)

Hirano, Seishiro (Tsukuba)

Horiguchi, Hyogo (Tochigi)

Ikeda, Masayuki (Kyoto)

Ikeda, Shunya (Otawara)

Imamura, Tomoaki (Nara)

Inaba, Ryoichi (Gifu)

Ishii, Akira (Nagoya)

Ishikawa, Sho (Tokyo)

Ito, Hiroto (Tokyo)

Kagamimori, Sadanobu (Toyama)

Katsuno, Shingo (Kato)

Katsuyama, Hironobu (Kurashiki)

Kawanishi, Masahiro (Suzuka)

Kikuchi, Shougo (Aichi)

Kishimoto, Takuji (Yonago)

Kishimoto, Tsuyoshi (Saitama)

Kobayashi, Nobumichi (Sapporo)

Koizumi, Akio (Kyoto)
Kondo, Naomi (Yamanashi)

Kumagai, Kazukiyo (Kashiwa)

Kunugita, Naoki (Kitakyusyu)

Kuriyama, Shinichi (Sendai)

Machida, Kazuhiko (Tokorozawa)

Masuda, Hiroshi (Nishinomiya)

Minami, Masayasu (Kawasaki)

Miyagawa, Muneyuki (Kawasaki)

Miyashita, Kazuhisa (Wakayama)

Moriyama, Masaki (Fukuoka)

Nagata, Chisato (Gifu)

Nakagawa, Hideaki (Ishikawa)

Nakahara, Daiichiro (Hamamatsu)

Nakaji, Shigeyuki (Hirosaki)

Nakamura, Kazutoshi (Niigata)

Nakamura, Keiko (Tokyo)

Nakamura, Kouichi (Tochigi)

Nakamura, Motoyuki (Morioka)

Nakao, Mutsuhiro (Tokyo)

Nakayama, Takeo (Kyoto)

Nasu, Tamie (Nagoya)

Nishinaga, Masanori (Nankoku)

Ogawa, Sumiko (Nago)

Ohira, Tetsuya (Suita)

Ohnishi, Mayumi (Nagasaki)

Ohno, Hideki (Mitaka)

Ojima, Toshiyuki (Hamamatsu)
Okamoto, Kazushi (Nagoya)

Okamura, Tomonori (Suita)

Ono, Masaji (Tsukuba)

Otsuki, Takemi (Kurashiki)

Ozasa, Kotaro (Kyoto)

Saijo, Yasuaki (Asahikawa)

Saito, Takeshi (Sapporo)

Sakai, Toshiyuki (Kyoto)

Sakata, Kiyomi (Morioka)

Sata, Fumihiro (Sapporo)

Sato, Tsutomu (Tokyo)

Shima, Masayuki (Nishinomiya)

Shiwaku, Kuninori (Izumo)

Sobue, Tomotaka (Tokyo)

Sugita, Minoru (Tokyo)

Takahashi, Masaya (Kawasaki)

Takebayashi, Toru (Tokyo)

Takeshita, Tatsuya (Wakayama)

Tanaka, Hideo (Nagoya)

Taoda, Kazushi (Otsu)

Tokudome, Shinkan (Nagoya)

Tominaga Suketami (Owariasahi)

Tsukamura, Hiroko (Nagoya)

Watanabe, Chiho (Tokyo)

Yanagi, U (Wako)

Yasuda, Nobufumi (Nankoku)

$\overline{1}$ In alphabetical order. 